The Influence of Postural Emotion Cues on Implicit Trait Judgements

\author{
Van Der Zant, T. ${ }^{1}$, Reid, J. ${ }^{1}$, Mondloch, C. J. ${ }^{2} \&$ Nelson, N. L. ${ }^{1}$ \\ The University of Queensland ${ }^{1}$ and Brock University ${ }^{2}$
}

Word Count: 4145

Address for correspondence:

*Tamara Van Der Zant, School of Psychology, The University of Queensland, St Lucia, QLD, 4067, Australia. Email: t.vanderzant@uq.edu.au 


\begin{abstract}
Perceptions of traits (such as trustworthiness or dominance) are influenced by the emotion displayed on a face. For instance, the same individual is reported as more trustworthy when they look happy than when they look angry. This overextension of emotional expressions has been shown with facial expression but whether this phenomenon also occurs when viewing postural expressions was unknown. We sought to examine how expressive behaviour of the body would influence judgements of traits and how sensitivity to this cue develops. In the context of a storybook, adults $(\mathrm{N}=35)$ and children (aged 5 to 8 years; $\mathrm{N}=60$ ) selected one of two partners to help face a challenge. The challenges required either a trustworthy or dominant partner. Participants chose between a partner with an emotional (happy/angry) face and neutral body or one with a neutral face and emotional body. As predicted, happy over neutral facial expressions were preferred when selecting a trustworthy partner and angry postural expressions were preferred over neutral when selecting a dominant partner. Children's performance was not adult-like on most tasks. The results demonstrate that emotional postural expressions can also influence judgments of others' traits, but that postural influence on trait judgments develops throughout childhood.
\end{abstract}

Keywords: Trustworthiness, dominance, facial expression, postural expression, children, traits 


\section{The Influence of Postural Emotion Cues on Implicit Trait Judgements}

From another's face we rapidly make judgements about how they feel, how they act, and who they are (Zebrowitz \& Montepare, 2008). These first impressions often include judgments of someone's age, gender, dominance, and trustworthiness and can have substantial impacts on our social interactions (Mileva, Cowan, Cobey, Knowles, \& Little, 2014; Oosterhof \& Todorov, 2009; Todorov, 2008; Todorov, Mende-Siedlecki, \& Dotsch, 2013; Zebrowitz \& Montepare, 2008).

First impressions judgments, particularly of trustworthiness and dominance, are closely tied to judgements of facial expressions. For example, adults judge facial expressions showing even slight anger to be more dominant and expressions showing even slight happiness to be more trustworthy (Mileva et al., 2014; Mondloch, Gerada, Proietti, \& Nelson, 2019; Oosterhof \& Todorov, 2009; Said, Sebe, \& Todorov, 2009; Todorov, 2008). This overgeneralization of facial expressions to judgments of other's traits, while made rapidly, are not generally accurate reflections of other's true characteristics (Todorov, 2017; Zebrowitz, 2017; Zebrowitz \& Montepare, 2008)

Recent research hints at the possibility that there is more to the link between emotional expressions and first impressions judgments than the simple overgeneralization of facial expression cues. It is possible that we overgeneralize emotion cues more broadly, such as linking vocal traits or body posture to the traits of others. For example, traits such as agreeableness and trustworthiness can be reliably identified from voice as well as postural gait, and postural expressions of dominance and submission are recognizable even when viewed for only 40ms (Rule, Adams, Ambady, \& Freeman, 2012; Schild, Stern, \& Zettler, 2019; Thoresen, Vuong, \& Atkinson, 2012). Thus, it is possible that postural emotional expressions in particular could be overgeneralized to trait judgments just as facial expressions are, although this has not yet been explored. 
Non-facial emotional cues like body posture also convey emotional information, occasionally more effectively than facial expressions (Aviezer, Trope, \& Todorov, 2012; de Gelder \& Van den Stock, 2011; Van Der Zant \& Nelson, 2020). This is particularly true for body cues related to aggression (Abramson, Marom, Petranker, \& Aviezer, 2017; Scrivner et al., 2019). Postural cues are also recognized early in childhood: 3- to 5-year-olds recognize emotion in body postures almost as early as they recognize emotion in facial expressions (Mondloch, Horner, \& Mian, 2013; Nelson \& Russell, 2011; Widen, 2013). In addition, children look more to the body than adults do (Nelson \& Mondloch, 2018, 2019), meaning that body posture may play a stronger role in children's judgments than they do in adult's judgments.

Children's first impressions are influenced by emotional cues such as facial features and emotional expressions. Like adults, children judge emotional faces as 'nice' or 'mean' (Cogsdill \& Banaji, 2015). Children also judge neutral faces with facial hair as 'dominant', but not trustworthy (Nelson, Dixson, \& Mondloch, 2019). Past research shows that children use facial expressions to make explicit judgments about others' traits: children judge happy faces as more trustworthy than angry faces (Caulfield, Ewing, Bank, \& Rhodes, 2016; Li, Heyman, Mei, \& Lee, 2019). However, when making implicit judgments, evidence that children consider emotional expressions is mixed. In an economic trust game, children associated smiling faces with trustworthiness (Ewing, Sutherland, \& Willis, 2019). In contrast, both when deciding with whom to share and in a storybook task where children selected partners to help with various challenges like fighting dragons or keeping secrets, children did not select smiling faces to help with trustworthiness challenges or angry faces to help with dominance challenges (Charlesworth, Hudson, Cogsdill, Spelke, \& Banaji, 2019; Mondloch, Gerada, Proietti, \& Nelson, 2019). That children are inconsistent in making implicit and explicit trait judgements from emotional faces leaves open the possibility that 
they are instead attending to non-facial emotion cues, such as body posture, a possibility that we explore in this research

In this study, we examined whether children and adults were more likely to select an emotional facial or an emotional postural expression when judging another's trustworthiness or dominance. We adapted the storybook task from Mondloch et al. (2019), in which participants read an adventure book where they confront a variety of challenges. For each challenge, participants are presented with pairs of people and they can choose which person of the pair they would like to help them overcome the challenge. Some of these challenges required a trustworthy partner and some required a dominant/strong partner. We selected this implicit measure of trait judgments because implicit measures most closely approximate these judgements in daily life (Mondloch, Gerada, Proietti, \& Nelson, 2019; Nelson, Dixson, \& Mondloch, 2019).

Past research has presented strong evidence that happy cues are associated with trustworthiness and anger cues are associated with dominance (e.g. Mondloch, Gerada, Proietti, \& Nelson, 2019; Oosterhof \& Todorov, 2009; Said, Sebe, \& Todorov, 2009). Thus, in this study, for trust challenges, participants were presented two potential partners, with one showing a happy face (paired with a neutral body) and one showing a happy body (paired with a neutral face). For dominance challenges, participants were presented one partner showing an angry face (paired with a neutral body) and one showing an angry body (paired with a neutral face). These incongruent stimuli allowed us to determine whether participants prefered happy facial or postural cues when judging another's trustworthiness, and whether particpiants preferred angry facial or postural cues when judging another's dominance.

Participant's selection of a partner showing an incongruent expression, for example, a happy face paired with a neutral posture, could be due to their preference for happy faces, or it could be due to their preference for neutral postures. To help differentiate between these 
two possibilities, we also incorporated trials where the potential partners showed either emotional or neutral cues. For trust challenges, participants were presented one partner showing a happy face and happy body and one partner showing a neutral face and neutral body. For dominance challenges, we incorporated angry cues rather than happy ones. These congruent stimuli allowed us to determine whether participants preferred emotional or neutral expressions when judging other's trustworthiness or dominance.

We expected that participants would be more likely to associate trustworthiness with happy facial expressions than happy postural expressions and would be more likely to associate dominance with angry postural expressions than angry facial expressions. We also anticipated that adults would be more likely than children to selectively choose happy faces to help in trust challenges and angry postures to help in dominance challenges.

\section{Method}

\section{Participants}

Participants were sixty children ( 28 females, $M_{\text {age }}=6.42$ years, range: $5.08-8.0$ years), recruited through a database of families willing to participate in developmental research with their children, and thirty-five adults ( 28 females, $M_{\text {age }}=21.10$, range: $17-50$ years) recruited from first year psychology courses. Participants received a small toy or course credit in exchange for participating. All protocols for the experiment received clearance from the University of Queensland Human Research Ethics Committee (18PSYCH-4-80-AH). Data for this study are available online at https://osf.io/5j927/.

\section{Materials}

Stimuli. Two Caucasian posers ( 1 male, 1 female) were drawn from the stimulus set used by Nelson and Mondloch (2017). Each poser contributed facial and postural expressions of anger, happiness, and neutrality. For each poser, two different postural expressions and two different facial expressions were selected for each emotion resulting in a total of 24 
unique expression components. The happy and angry facial and postural expressions were validated in previous research, with recognition rates above $71^{1 \%}$ for each expression (Nelson \& Mondloch, 2017). In addition, we also assessed the recognizability of the neutral stimuli presented in this study. Data from an additional sample of 29 children $\left(M_{\text {Age }}=6.99\right.$ years, range: 5.0-10.5 years) showed that neutral stimuli were well recognized as 'neutral' when participants selected from three possible labels (angry, happy, and neutral). For these stimuli, $84.5 \%$ recognized the neutral faces and $75 \%$ recognized the neutral bodies.

Using Adobe Photoshop, the 24 expression components were used to create two sets of stimuli: incongruent and congruent expressions. Congruent stimuli showed the same emotion in the facial and postural expression and showed happy, angry, or neutral expressions. Incongruent stimuli showed different emotions in the facial and postural expression, with one cue being neutral and one being emotional (happy or angry). Thus, neutral faces were paired with emotional bodies and emotional faces were paired with neutral bodies. A total of 28 unique images (16 incongruent and 12 congruent) were created (See Supplemental Materials for more information).

Participants viewed pairs of images of either the male or female poser, side-by-side on a white background (see Figure 1). Within the image pairs, the emotional cue was counterbalanced between left and right side, and image resolution was $700 \times 540 \mathrm{px}$. The incongruent stimuli image pairs consisted of a happy face on a neutral body paired with a neutral face on a happy body or an angry face on a neutral body paired with a neutral face on an angry body. The congruent stimuli image pairs consisted of an angry face and body paired with a neutral face and body or a happy face and body paired with a neutral face and body. In total, we created 32 image pairs (half with the male poser, half with the female poser). Of

\footnotetext{
${ }^{1}$ In the earlier validation study by Nelson \& Mondloch, the male angry facial expression was recognized by $71 \%$ of participants. All other expressions used in this study were recognized by more than $71 \%$ of participants.
} 
these image pairs, 16 showed congruent expressions and 16 showed incongruent expressions, and half of the image pairs showed happy cues and half showed angry cues. This resulted in 8 Incongruent Happy pairs, 8 Incongruent Angry pairs, 8 Congruent Happy pairs, and 8 Congruent Angry pairs.

A)

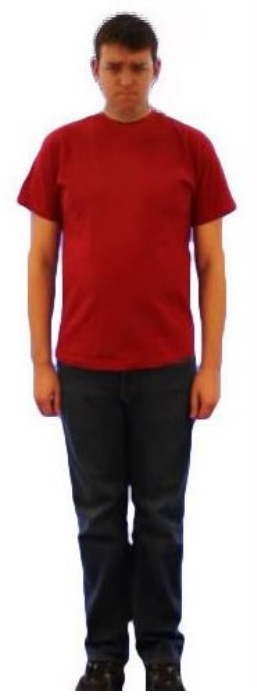

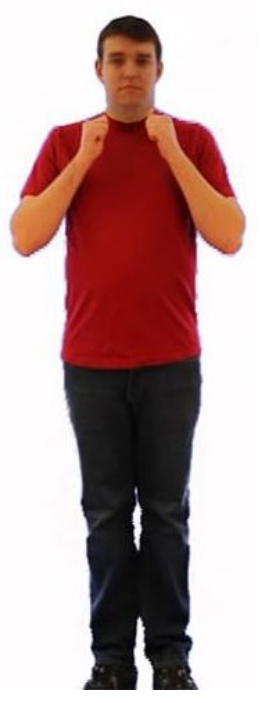

B)

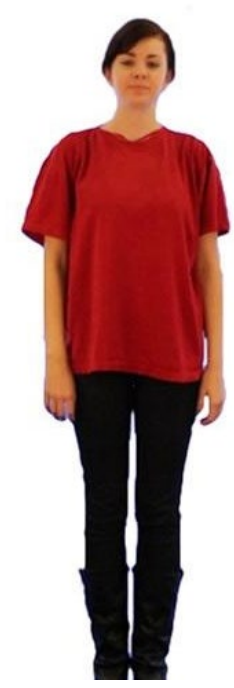

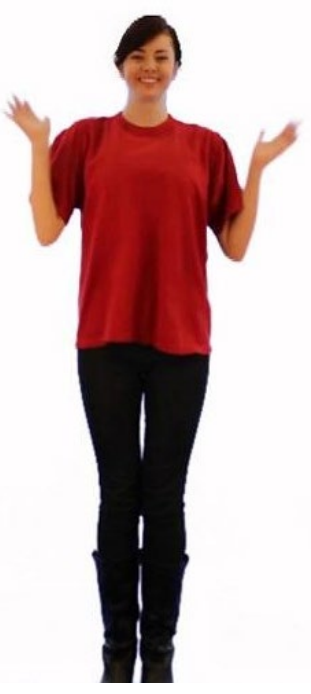

Figure 1. Examples of incongruent and congruent stimuli pairs. A) shows an angry incongruent stimulus pair (angry face on left, angry body on right). B) shows a happy congruent stimulus pair (neutral face and body on left, happy face and body on right). Written consent for publication of images obtained from both posers.

Procedure. Participants first completed the storybook task, followed by an explicit emotion recognition task.

Storybook Task. The storybook task was drawn from Mondloch et al. (2019). The story asked participants to imagine themselves on a magical journey to a treasure-filled island. Participants were told that they would face many obstacles on their adventure and that they could enlist the help of one of two twins. Adult participants read through the storybook and answered the questions on their own. Child participants were read the story by the experimenter and indicated their responses on a tablet by tapping the screen.

Participants were presented with six challenges in which an ideal partner would be trustworthy (e.g., would keep a secret) and six in which an ideal partner would be 
dominant/strong (e.g., would help fight a dragon). After hearing each challenge, two image pairs of the same identity were presented one at a time (Figure 1). The first pair of partners participants could choose from was an incongruent pair in which one partner had an emotional face with neutral body and the other had a neutral face with an emotional body. The emotion conveyed by the two partners was matched within each trial. The second pair of partners participants chose from was a congruent pair in which of one partner had a neutral face and body and one partner had an emotional face and body. The emotion displayed in both trials matched the trait being assessed in the scenario; happy expressions were shown for trustworthy challenges and angry expressions were shown for dominance challenges.

Two versions of the task were created to control for potential gender effects. Gender of the poser alternated for each question and this order was reversed between the two forms. This was to account for any effect that gender may have on the particular question being asked. In each form of the task there was three blocks. In Blocks 1 and 3, participants indicated which twin they wanted to help them and in Block 2, they indicated which twin they wanted to disappear so the remaining twin would help them. The reverse-scoring of Block 2 ensured that children did not simply choose the most emotional poser for each trial. In total, participants completed six trustworthy and six dominance scenarios, each followed by one incongruent and one congruent image pair.

Emotion recognition task. Following the completion of the storybook task, participants next judged the emotion being felt by each twin they met on the island. Participants viewed the 28 unique individual stimulus images one at a time and were asked “How do you think this person is feeling: angry, happy, or neutral?”. Children then selected the emotion label they thought best fit the expression.

\section{Results}




\section{Implicit Trait Judgement Task}

Responses in Comparison to Chance. We first conducted a series of one-sample ttests to determine whether participants' selection of partners in the challenges was different from chance responding (set at .50) across the stimulus types and age groups $(\alpha=.05 / 16=$ .003). Full t-test tables can be found in the Supplemental Materials. For incongruent stimuli (emotional faces vs. emotional bodies), we found that across the whole sample, participants selected the partner with the emotional face more often than would be expected by chance responding for trust challenges and less often than would be expected by chance for dominance challenges $(p \mathrm{~s}<.001)$. This pattern was replicated across both adults and children ( $p$ s $<.001)$. For congruent stimuli (emotional vs. neutral expressions), we found that across the whole sample, participants selected the emotional partner more often than would be expected by chance for both trust and dominance challenges $(p \mathrm{~s}<.001)$. For dominance challenges, this pattern was replicated across both adults and children $(p s<.003)$. However, for trust challenges, adults were more likely to select the emotional partner $(p=.002)$, but children were not $(p=.061)$.

These results indicate that all age groups were sensitive to facial and postural cues when selecting a dominant partner, with postural cues having the larger influence. However, only adults were sensitive to facial and postural cues when selecting a trustworthy partner, with facial cues having the larger influence.

Developmental Differences in Responding for Incongruent Stimuli. To determine whether participants' selection of emotion cues varied with the challenges they encountered, we conducted a 2 (trait: trustworthy, dominance) $\times 2$ (age: children, adults) mixed ANOVA. The DV was the proportion of trials in which the partner showing the emotional face was chosen. For all analyses, Greenhouse-Geisser corrections were applied to address violations 
of sphericity and all effects were followed up with Bonferroni-corrected post hoc comparisons.

The main effect of trait, $F(1,92)=233.61, p<.001, \eta^{2}=.54$, indicated that participants chose the partner with the emotional face (rather than the emotional body) more often for trustworthy challenges $(M=.71)$ than for dominance challenges $(M=.21)$. The main effect of age, $F(1,92)=5.69, p=.019, \eta^{2}=.01$, showed that adults $(M=.51)$ were more likely to choose the emotional face than children were $(M=.43)$. The trait $\mathrm{x}$ age interaction, $F(1,92)=4.59, p=.035, \eta^{2}=.01$ showed that, for trust challenges, adults $(M=$ .81) were more likely to choose the emotional face than children were $(M=.65), p=.002(\alpha$ $=.05 / 4=.013)($ Figure 2). However, for dominance challenges, there were no differences between age groups $(p \mathrm{~s}=.825)$.

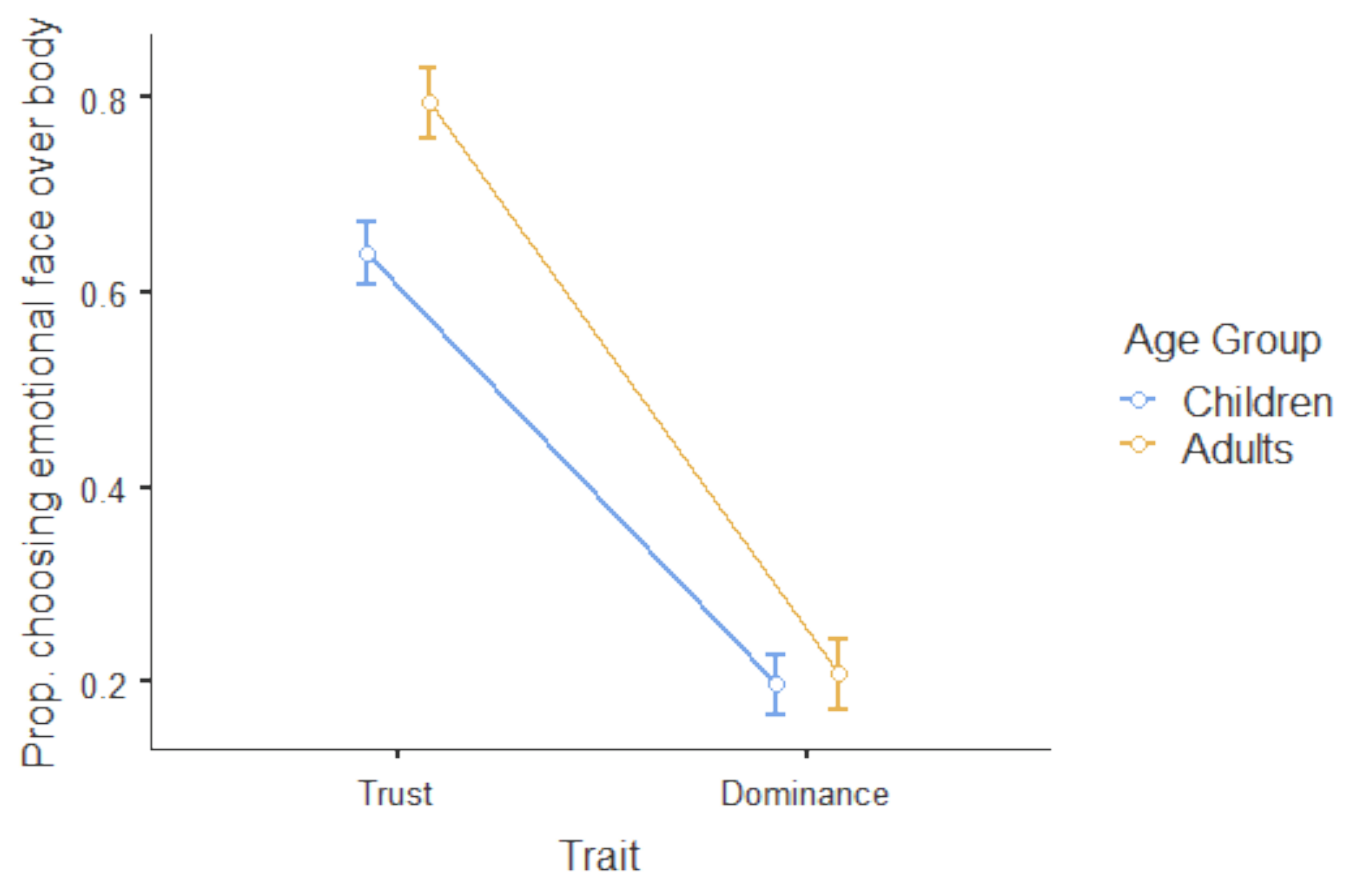

Figure 2. Proportion of trials in which participants chose the emotional face for the incongruent stimuli as a function of trait and age. Error bars represent standard errors.

Developmental Differences in Responding for Congruent Stimuli. To examine whether participants' selection of emotional vs. non-emotional partners varied across challenges, we conducted a 2 (trait: trustworthy, dominance) $\times 2$ (age: children, adults) mixed 
ANOVA. The DV was the proportion of trials in which the emotional, rather than neutral, partner was chosen.

The main effect of trait, $F(1,92)=4.24, p=.042, \eta^{2}=.02$, indicated that, overall, participants chose the emotional partner (rather than the neutral partner) more often for dominance challenges $(M=.72)$ than for trustworthy ones $(M=.61)$. There was no effect of age, $F(1,92)=0.14, p=.708, \eta^{2}=.00$. There was, however, a Trait $\times$ Age interaction, $F(1$, $92)=7.05, p=.009, \eta^{2}=.03$, which showed that children chose the emotional partner more often for dominance challenges $(M \mathrm{~s}=.76)$ than for trustworthy ones $(M \mathrm{~s}=.57), p \mathrm{~s}<.001$ (Figure 3). However, adults were similarly likely to select the emotional partner for trustworthy $(M=.66)$ and dominance challenges $(M=.64), p=.709$.

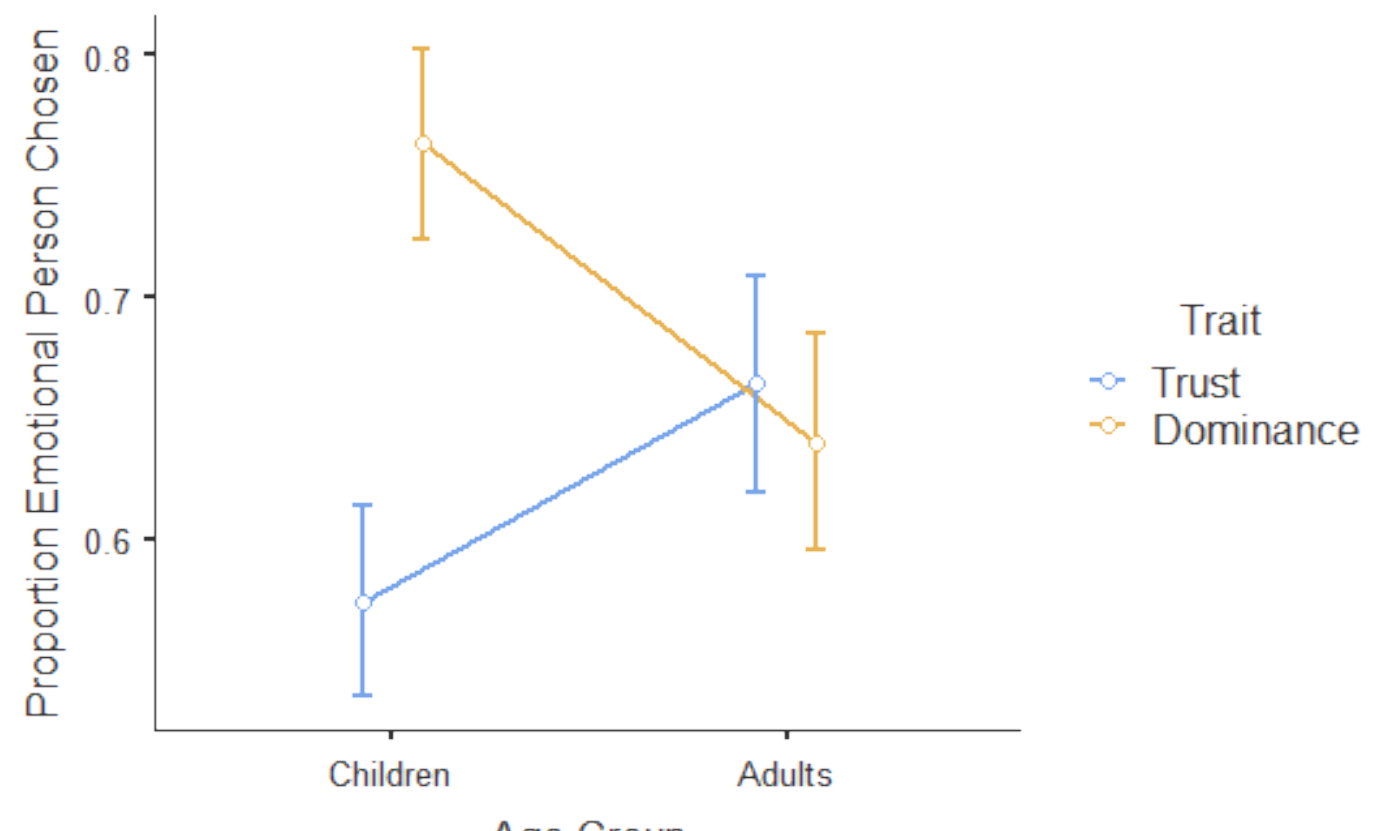

Age Group

Figure 3. Proportion of trials in which participants chose the emotional person for the congruent stimuli as a function of trait and age. Error bars represent standard errors.

\section{Explicit Judgment Task}

Incongruent stimuli. To examine participants' judgments of the emotion shown in the incongruent stimuli, we conducted a 2 (emotion: happy, angry) $\times 2$ (cue: facial, postural) 
$\times 2$ (age: children, adults) mixed ANOVA. The DV was the proportion of stimuli participants labelled as the expected emotion (happy or angry).

There was a main effect of cue, $\mathrm{F}(1,92)=668.56, \mathrm{p}<.001, \mathrm{n} 2=.565$, but no main effect of emotion, $\mathrm{F}(1,92)=3.66, \mathrm{p}=.059, \mathrm{n} 2=.00$. These were superseded by an emotion $\times$ cue interaction, $F(1,92)=132.65, p<.001, \eta^{2}=.09$ which showed that participants were more likely to recognize happy faces than angry faces, $p<.001$. In contrast, participants were more likely to recognize angry bodies than happy bodies, $p<.001,(\alpha=.05 / 2=.025)$. The Cue $\times$ Age interaction, $F(1,92)=7.07, p=.009, \eta^{2}=.01$, showed that although children and adults were both more likely to recognize the emotion in face than body expressions, this difference was larger for adults than children $p \mathrm{~s}<.001,(\alpha=.05 / 2=.025)$ (Figure 4). A confusion matrix detailing participants' specific responses can be found in the Supplemental Materials.

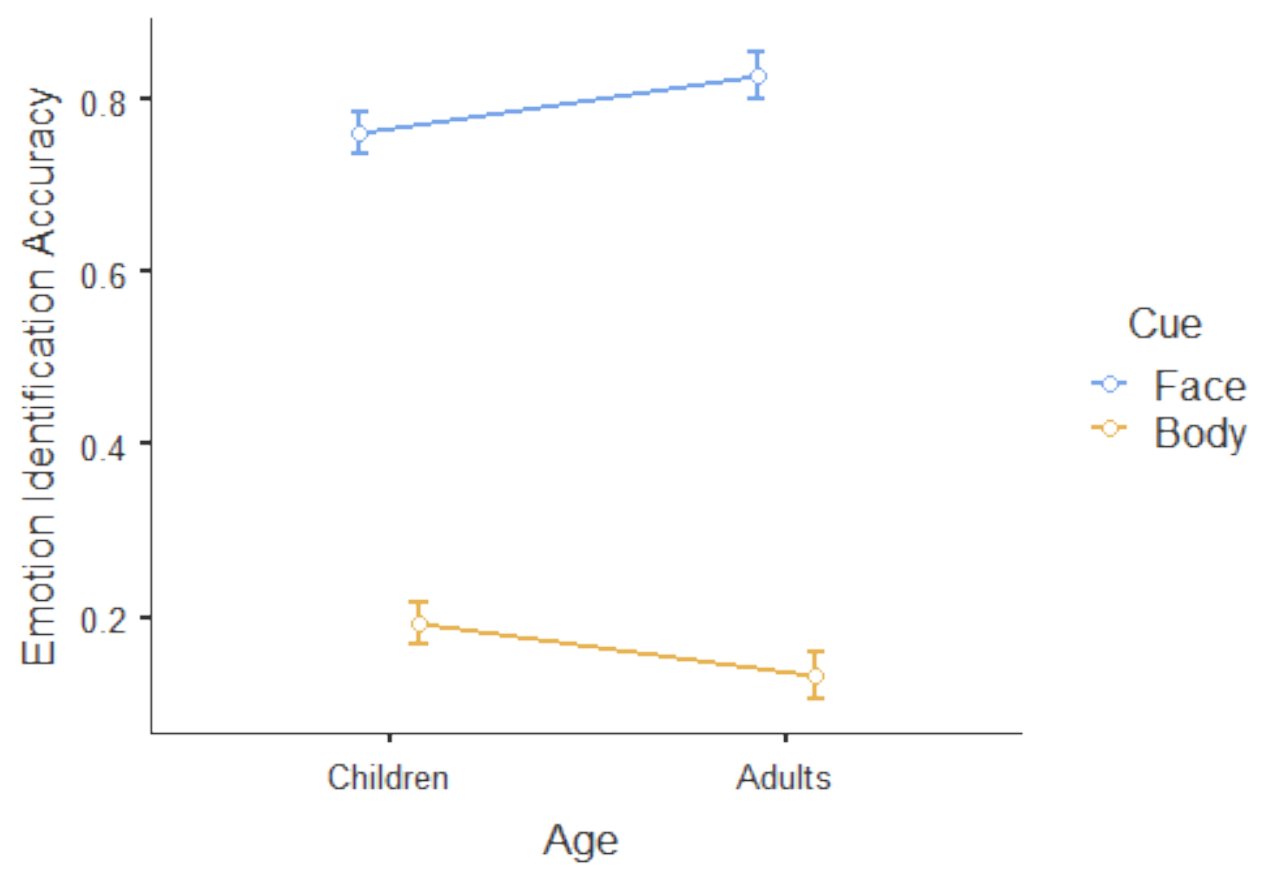

Figure 4. Proportion of incongruent stimuli judged as showing the expected emotion, presented by age group and emotional cue. Error bars represent standard errors. 
Congruent stimuli. To examine participants' judgments of the congruent stimuli, a 2 (emotion: happy, angry) $\times 2$ (age: children, adults) mixed ANOVA was conducted. The DV was the proportion of stimuli participants labelled as the expected emotion (happy or angry).

The main effects of emotion, $F(1,92)=60.90, p<.001, \eta^{2}=.19$, and age, $F(1,92)=$ 8.01, $p=.006, \eta^{2}=.04$, were superseded by an Emotion $\times$ Age interaction, $F(1,92)=14.3, p$ $<.001, \eta^{2}=.04$ (Figure 5). For happy stimuli, adults $(M=.94)$, and children $(M=.95)$ were similarly likely to label the expressions as expected, $p=.866$. For angry stimuli, however, adults $(M=.84)$ were more likely to label the emotion as expected than children $(M=.64), p$ $<.001$.

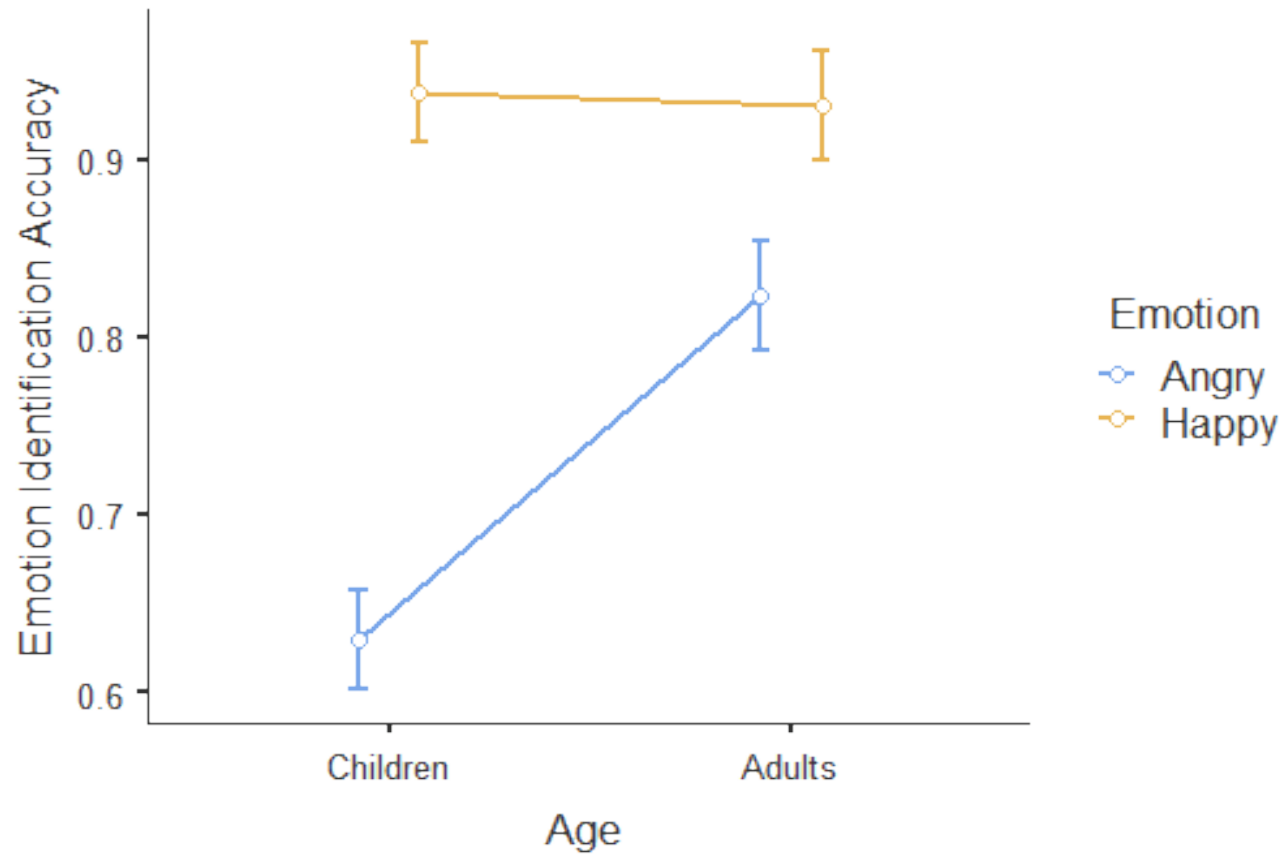

Figure 5. Proportion of trials in which participants identified the expected emotion in congruent stimuli as a function of age and emotion. Error bars represent standard errors.

\section{Discussion}

This study is the first to show that adults' and children's first impressions of others can be influenced by emotional body postures as well as emotional facial expressions. These 
findings contribute to a growing literature elucidating the development of trait judgments. Previous literature has found clear evidence that children associate happy facial expressions with trustworthiness (e.g. Caulfield, Ewing, Bank, \& Rhodes, 2016; Ewing, Sutherland, \& Willis, 2019; Li, Heyman, Mei, \& Lee, 2019), but evidence that children associate angry facial expressions with dominance has been inconclusive (e.g. Charlesworth, Hudson, Cogsdill, Spelke, \& Banaji, 2019; Mondloch et al., 2019). The findings of this study suggest that children may be more likely to associate angry postural expressions with dominance than angry facial expressions, perhaps explaining the inconsistency of previous research.

Participants generally associated dominance with anger displayed in the body more than with anger displayed in the face. In addition, for congruent stimuli participants selected angry partners vs. neutral ones, suggesting that when participants selected the angry body paired with a neutral face for the incongruent stimuli, they were attending to the angry body rather than the neutral face. Similarly, trust was generally associated with happiness displayed in the face more than with happiness displayed in the body. And, for the congruent stimuli, participants selected the happy partner over the neutral one, suggesting that when viewing the incongruent stimuli, participants were attending to the happy face rather than the neutral body. These findings indicate that the link between judging others' emotions and traits is not simply due to the overlap of facial structural elements and emotional expression movements. Instead, it appears that the overlap between judgements of emotions and traits is linked to the overextension of the emotion concept itself.

When presented congruent stimuli pairs, where one partner had an emotional face and body and one partner had a neutral face and body, participants were more likely to select an angry partner for a dominance challenge than a happy partner for a trust challenge. However, this effect was driven entirely by children: adults were similarly likely to select an emotional person for dominance and trust trials. Children's lower likelihood of selecting a happy person 
for trust challenges wasn't attributable to their inability to recognize the stimuli presented, as shown by their adult-like recognition of the expressions in the explicit judgement task. This pattern of results was also was not due to children misattributing emotion to the neutral faces, as shown by the high recognizability of the neutral stimuli used in this task. When children judged trust from the congruent stimuli pairs, however, their responses were not different from chance responding, suggesting that children may not judge happy people to be more trustworthy than neutral people, consistent with results from Mondloch et al. (2019).

When presented incongruent stimuli pairs where one partner had an emotional face/neutral body and one partner had a neutral face/emotional body, participants were more likely to select a partner with an emotional face for a trust challenge than a dominance challenge. However, there were developmental differences across the traits presented. For dominance challenges children's responses were adult-like, but for trust challenges they were less likely to select partners with emotional faces than were adults. Children did select emotional faces for trust challenges more often than would be expected by chance, suggesting that children do attend to facial expression cues more than bodily expression cues when selecting a trustworthy partner, just not as much as adults do.

Future research may expand on the current study in various ways. This study focussed on the association between anger and happiness with dominance and trust, respectively, as these are two emotion-trait relationships that are found to be robust. However, other combinations, such as the relationship between fear expressions and vulnerability/shyness in child posers could provide additional avenues to assess the role of face and body cues of emotion in indicating traits (Collova, Sutherland \& Rhodes, 2019; Zebrowitz \& Montepare, 2008). Additionally, as happiness is typically poorly recognised in bodies (de Gelder \& Van den Stock, 2011), the inclusion of other emotional cues - for example vocal cues to happiness - may provide different patterns of results (e.g. Morton, Trehub, \& Zelazo, 2003; Vidas, 
Dingle, \& Nelson, 2018). A limitation of the current study was use of a non-orthogonal design, where happy cues were paired only with trustworthy challenges and anger cues were paired only with dominance questions. Future research may consider pairing happy cues with dominance questions, for example, to determine how various emotion expressions influence trait judgements. Finally, incorporating dynamic, genuine (i.e. not posed), may increase the ecological validity of future research, providing insight into how trait judgements influence daily interactions (Van Der Zant \& Nelson, 2020).

\section{Conclusion}

The finding that happy faces influenced judgements of trust, and angry bodies influenced judgements of dominance, provides initial evidence of variation in how emotion expression may alter judgements of traits dependent on which cues (face and/or body) are available. Research focussed exclusively on facial expressions as a means of influencing trait judgments fails to account for the influence of other cues that can factor into these judgments, providing an incomplete understanding of this phenomenon. 


\section{References}

Abramson, L., Marom, I., Petranker, R., \& Aviezer, H. (2017). Is fear in your head? A comparison of instructed and real-life expressions of emotion in the face and body. Emotion, 17(3), 557-565. https://doi.org/10.1037/emo0000252

Aviezer, H., Trope, Y., \& Todorov, A. (2012). Body cues, not facial expressions, discriminate between intense positive and negative emotions. Science, 338(November 2012), 1225-1230. Retrieved from https://www.semanticscholar.org/paper/AviezerPositive-and-Negative-Emotions-Body-Cues-\%2C-RanaKakuda/6101d0fd6f00679d56b60c12d4f97377ebc29cf6

Caulfield, F., Ewing, L., Bank, S., \& Rhodes, G. (2016). Judging trustworthiness from faces: Emotion cues modulate trustworthiness judgments in young children. British Journal of Psychology (London, England: 1953), 107(3), 503-518.

https://doi.org/10.1111/bjop.12156

Charlesworth, T. E. S., Hudson, S. kiera T. J., Cogsdill, E. J., Spelke, E. S., \& Banaji, M. R. (2019). Children use targets' facial appearance to guide and predict social behavior. Developmental Psychology, 55(7), 1400-1413. https://doi.org/10.1037/dev0000734

Cogsdill, E. J., \& Banaji, M. R. (2015). Face-trait inferences show robust child-adult agreement: Evidence from three types of faces. Journal of Experimental Social Psychology, 60, 150-156. https://doi.org/10.1016/j.jesp.2015.05.007

Collova, J. R., Sutherland, C. A., \& Rhodes, G. (2019). Testing the functional basis of first impressions: Dimensions for children's faces are not the same as for adults' faces. Journal of personality and social psychology, 117(5), 900.

de Gelder, B., \& Van den Stock, J. (2011). The bodily expressive action stimulus test (BEAST). Construction and validation of a stimulus basis for measuring perception of whole body expression of emotions. Frontiers in Psychology, 2(AUG), 1-6. 
https://doi.org/10.3389/fpsyg.2011.00181

Ewing, L., Sutherland, C. A. M., \& Willis, M. L. (2019). Children show adult-like facial appearance biases when trusting others. Developmental Psychology, 55(8), 1694-1701. https://doi.org/10.1037/dev0000747

Li, Q., Heyman, G. D., Mei, J., \& Lee, K. (2019). Judging a book by Its cover: Children's facial trustworthiness as judged by strangers predicts their real-world trustworthiness and peer relationships. Child Development, 90(2), 562-575.

https://doi.org/10.1111/cdev.12907

Mileva, V. R., Cowan, M. L., Cobey, K. D., Knowles, K. K., \& Little, A. C. (2014). In the face of dominance: Self-perceived and other-perceived dominance are positively associated with facial-width-to-height ratio in men. Personality and Individual Differences, 69, 115-118. https://doi.org/10.1016/j.paid.2014.05.019

Mondloch, C. J., Gerada, A., Proietti, V., \& Nelson, N. L. (2019). The influence of subtle facial expressions on children's first impressions of trustworthiness and dominance is not adult-like. Journal of Experimental Child Psychology, 180, 19-38. https://doi.org/10.1016/j.jecp.2018.12.002

Mondloch, C. J., Horner, M., \& Mian, J. (2013). Wide eyes and drooping arms: Adult-like congruency effects emerge early in the development of sensitivity to emotional faces and body postures. Journal of Experimental Child Psychology, 114(2), 203-216. https://doi.org/10.1016/j.jecp.2012.06.003

Morton, J. B., Trehub, S. E., \& Zelazo, P. D. (2003). Sources of inflexibility in 6-year-olds' understanding of emotion in speech. Child Development, 74(6), 1857-1868. https://doi.org/10.1046/j.1467-8624.2003.00642.x

Nelson, N. L., Dixson, B. J. W., \& Mondloch, C. J. (2019). The influence of beards on children's first impressions: Trait labels vs. behavioural judgements, Under Review . 
Nelson, N. L., \& Mondloch, C. J. (2017). Adults' and children's perception of facial expressions is influenced by body postures even for dynamic stimuli. Visual Cognition, 1-12. https://doi.org/10.1080/13506285.2017.1301615

Nelson, N. L., \& Mondloch, C. J. (2018). Children's visual attention to emotional expressions varies with stimulus movement. Journal of Experimental Child Psychology, 172, 13-24. https://doi.org/10.1016/j.jecp.2018.03.001

Nelson, N. L., \& Mondloch, C. J. (2019). Children's perception of emotions in the context of live interactions: Eye movements and emotion judgements. Behavioural Processes, 164, 193-200. https://doi.org/10.1016/j.beproc.2019.05.006

Nelson, N. L., \& Russell, J. A. (2011). Preschoolers' use of dynamic facial, bodily, and vocal cues to emotion. Journal of Experimental Child Psychology, 110(1), 52-61. https://doi.org/10.1016/j.jecp.2011.03.014

Oosterhof, N. N., \& Todorov, A. (2009). Shared perceptual basis of emotional expressions and trustworthiness impressions from faces. Emotion, 9(1), 128-133. https://doi.org/10.1037/a0014520

Said, C. P., Sebe, N., \& Todorov, A. (2009). Structural resemblance to emotional expressions predicts evaluation of emotionally neutral faces. Emotion, 9(2), 260-264. https://doi.org/10.1037/a0014681

Schild, C., Stern, J., \& Zettler, I. (2019). Linking men's voice pitch to actual and perceived trustworthiness across domains. Behavioral Ecology, 31, 164-175. https://doi.org/10.1093/beheco/arz173

Scrivner, C., Choe, K. W., Henry, J., Lyu, M., Maestripieri, D., \& Berman, M. G. (2019). Violence reduces attention to faces and draws attention to points of contact. Scientific Reports, 9(1), 1-8. https://doi.org/10.1038/s41598-019-54327-3

Thoresen, J. Vuong, A. \& Atkinson, A. (2012). First impressions: Gait cues drive reliable 
trait judgements. Cognition, 124(3), 261-271.

https://doi.org/10.1016/j.cognition.2012.05.018

Todorov, A. (2008). Evaluating faces on trustworthiness: An extension of systems for recognition of emotions signaling approach/avoidance behaviors. Annals of the New York Academy of Sciences, 1124, 208-224. https://doi.org/10.1196/annals.1440.012

Todorov, A. (2017). Face Value. New Jersey: Princeton University Press.

Todorov, A., Mende-Siedlecki, P., \& Dotsch, R. (2013). Social judgments from faces. Current Opinion in Neurobiology, 23(3), 373-380. https://doi.org/10.1016/j.conb.2012.12.010

Van Der Zant, T. \& Nelson, N. L. (2020). Motion increases recognition of spontaneous postures but not facial expressions. [Manuscript submitted for publication].

Vidas, D., Dingle, G. A., \& Nelson, N. L. (2018). Children's recognition of emotion in music and speech. Music \& Science, 1, 205920431876265.

https://doi.org/10.1177/2059204318762650

Widen, S. C. (2013). Children's interpretation of facial expressions: The long path from valence-based to specific discrete categories. Emotion Review, 5(1), 72-77. https://doi.org/10.1177/1754073912451492

Zebrowitz, L. A. (2017). First Impressions From Faces. Current Directions in Psychological Science, 26(3), 237-242. https://doi.org/10.1177/0963721416683996

Zebrowitz, L. A., \& Montepare, J. M. (2008). Social Psychological Face Perception: Why Appearance Matters. Social and Personality Psychology Compass, 2(3), 1497-1517. https://doi.org/10.1111/j.1751-9004.2008.00109.x 